\title{
Accretion onto Black Holes and Neutron Stars: Differences and Similarities
}

\author{
Rashid Sunyaev \\ 1 Max-Planck-Institut für Astrophysik, Garching, Germany \\ 2 Space Research Institute, Moscow, Russia
}

\begin{abstract}
Accreting black holes and neutron stars at luminosities above 0.01 of the critical Eddington luminosity have a lot of similarities, but also drastic differences in their radiation and power density spectra. The efficiency of energy release due to accretion onto a rotating neutron star usually is higher than in the case of a black hole. The theory of the spreading layer on the surface of an accreting neutron star is discussed. It predicts the appearance of two bright belts equidistant from the equator. This layer is unstable and its radiation flux must vary with high frequencies.
\end{abstract}

\section{Introduction}

One of the most important properties of accreting black holes in our Galaxy was discovered by Riccardo Giacconi and the Uhuru Team in 1971, when they discovered the spectral transition of Cyg X-1 from the soft to the hard state (Tananbaum et al. 1972). Simultaneously, a radio source appeared in the vicinity of $\mathrm{Cyg} \mathrm{X}-1$. Radio observations permitted its localization with high accuracy and the identification of the X-ray source with a bright star of the 9 th magnitude. Immediately thereafter, measurements of its optical spectrum showed that this star is member of a 5.6-day non-eclipsing binary with an optically invisible companion (Bolton 1972). Lyuty et al. (1973) interpreted the observed ellipsoidal variations in the brightness of the optical star as a result of the gravitational influence of a nearby black hole invisible in optical light. Today Cyg X-1 is the best-known steadily accreting black hole in our Galaxy. Now we have a list with more than 12 excellent black-hole candidates and many of them show similar soft- to hard state transitions (Tanaka \& Shibazaki 1996). Recently, Cyg X-1 experienced the third transition from a hard to a soft state in 18 years (Fig. 1).

Such transitions became a signature of black holes. Today we know that all galactic black-hole candidates show a very soft X-ray spectrum. As predicted by standard accretion theory, this is a multicolor disk spectrum (cf. Shakura \& Sunyaev 1973) or a power-law hard X-ray spectrum with a Wien-type decay at high energies formed due to comptonization (Sunyaev \& Trümper 1979, Sunyaev \& Titarchuk 1980). Sometimes we do not even see the high frequency decay yet. Therefore, usually when a newly discovered X-ray transient shows an extremely hot tail in its X-ray spectrum, we immediately refer to it as a black-hole candidate. 


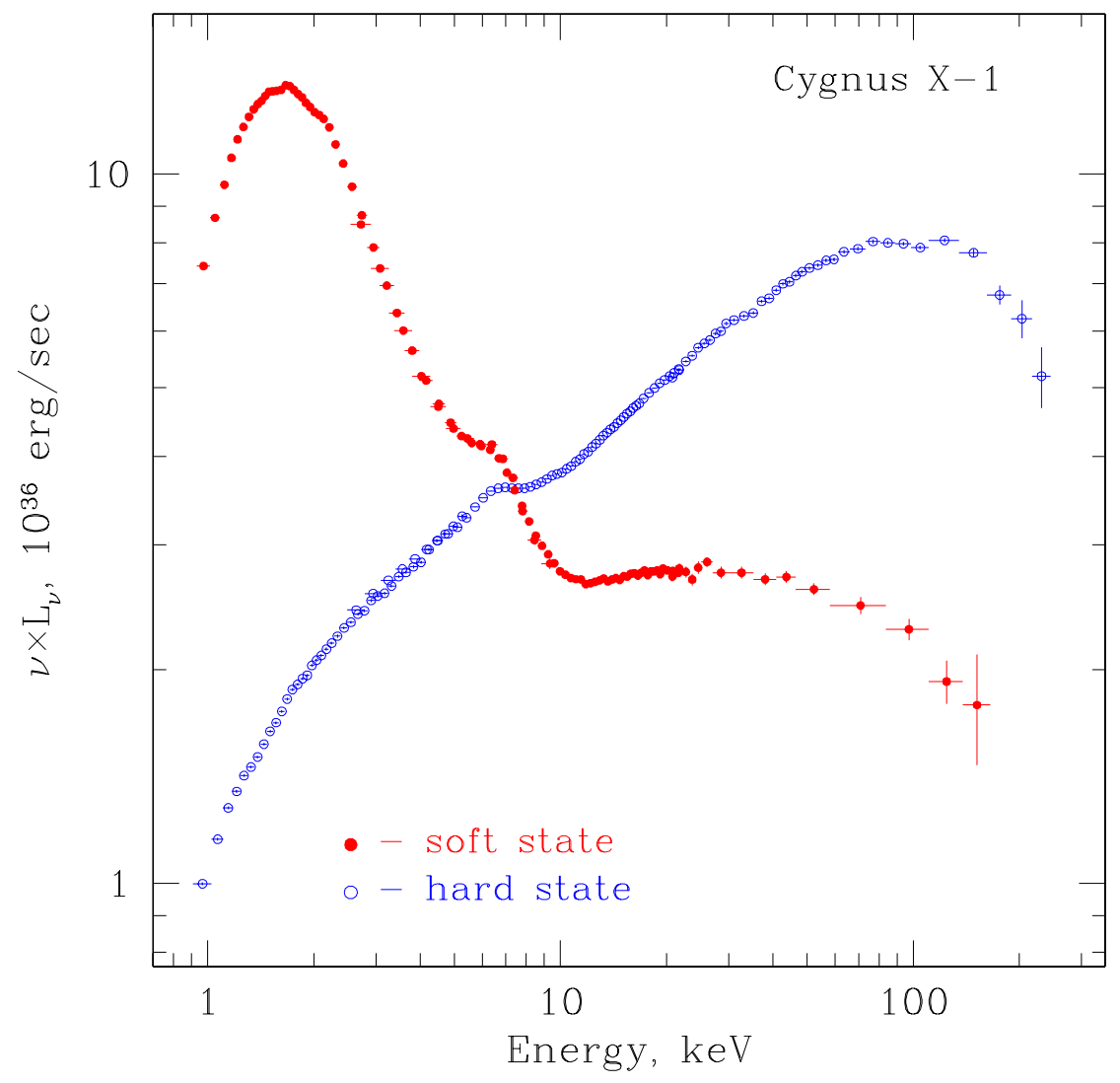

Fig. 1. The spectral energy distribution of Cyg X-1 in the soft (filled circles) and hard (open circles) spectral state. Data are shown of nearly simultaneous ASCA and RXTE observations on March 26, 1996 (hard state) and May 30, 1996 (soft state); cf. Gilfanov, Churazov \& Revnivtsev (2000).

Neutron stars without magnetic fields and black holes have practically the same gravitational potential and must show many similarities. Nevertheless, we know now that they have very different X-ray spectra and variability characteristics. One of the great surprises of the last 15 years of observations is the discovery that neutron stars also exhibit soft- to hard-state transitions (Fig. 2).

Neutron stars with small magnetic fields usually have spectra which are significantly harder than the spectra of multicolor accretion disks around black-hole candidates in a high/soft state. But their spectra are usually much softer than the spectra of black-hole candidates in the hard/low state. Sometimes we observe hot tails in the persistent flux of X-ray bursters. However, 


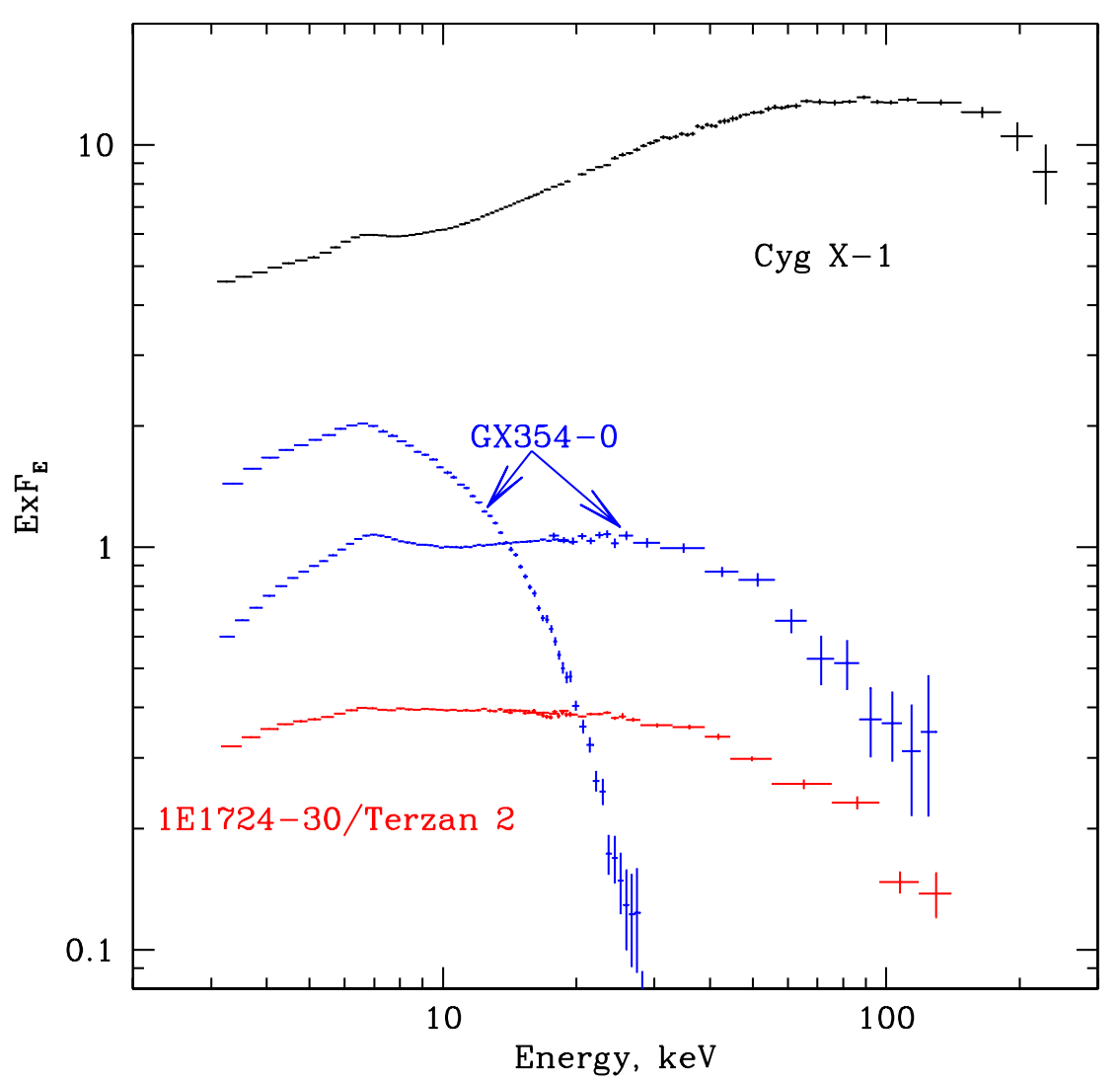

Fig. 2. The energy spectra of three X-ray binaries. The neutron star in GX354-0 (4U1728-34) is shown in two spectral states - low/hard and high/soft. Even in the hard state the neutron-star spectra are much softer than the spectrum of Cyg X-1 (accreting black hole). Adapted from Revnivtsev \& Sunyaev (2000).

spectra of these hot tails from neutron stars are much steeper than in the case of black holes and contain a smaller fraction of the source luminosity. It seems that now we know the reason. In the case of black-hole accretion we only see the radiation of accretion disk - plus, maybe, the corona above it (Galeev et al. 1979) or the advection flow with even smaller accretion efficiency (Narayan \& Yi 1995). In the case of neutron stars we have an object with a solid surface. Therefore, part of the gravitational energy of the accreting matter must be released in an extended accretion disk, and another 
part in the narrow boundary layer in the vicinity of the neutron star where accreting matter is decelerating from the Keplerian velocity (of the order of half the velocity of light) to the velocity of rotation at the equator of the neutron star. The surface of the star is able to produce enough soft protons for comptonization to cool down the hot parts of the disk and boundary layer to temperatures below $20 \mathrm{keV}$ (Sunyaev \& Titarchuk 1989). The physics of the boundary layer permits us to explain the strong differences between the radiation spectra of accreting black holes and neutron stars. It also predicts a strong difference in the characteristic variability timescales of the X-ray flux from black holes and neutron stars (see below).

\section{Efficiency of Accretion onto a Rapidly Rotating Neutron Star}

The recent discovery of quasi-periodic oscillations (QPO) with frequencies of the order of 500-600 Hz during the nuclear bursts on the surface of a neutron star appears to be very strong evidence of neutron-star rotation with the same frequency, or with periods of the order of 1.6-2 ms (Strohmayer et al. 1998). This interpretation is natural for a nuclear burning front propagating on the surface of a rapidly rotating neutron star. A bright front region manifests itself as a hot spot giving rise to the QPO. It is important that for a given neutron star the QPO frequency remains the same from burst to burst.

The efficiency of accretion onto neutron stars is higher (usually) than the efficiency of accretion onto black holes. The reason is obvious: in the case of a black hole we have an event horizon and an effective energy release and the release of the observed radiation flux might occur only in the accretion flow well beyond the event horizon. In the case of a neutron star without a strong magnetic field part of the energy is released in the extended accretion disk and another part is liberated in the narrow boundary layer near the surface of the neutron star. In Newtonian mechanics energy release in the boundary layer is equal to

$$
L_{s}=\frac{1}{2} \frac{G M \dot{M}}{R_{*}}\left(1-\frac{f}{f_{k}}\right)^{2},
$$

or is equal to the energy liberated in the disk

$$
L_{d}=\frac{1}{2} \frac{G M \dot{M}}{R_{*}}
$$

in the case of a slowly rotating compact star. Here and below $M$ is the gravitational mass of the star, $R_{*}$ is its radius, $f_{*}=\frac{1}{2 \pi} \sqrt{\frac{G M}{R_{*}^{3}}}$ the cyclic keplerian frequency near the its surface, $f$ is the frequency of stellar rotation and $\dot{M}$ is the accretion rate.

The problem becomes much more complicated in the case of General Relativity. Kerr metrics is not applicable to the case of rapidly rotating neutron 
star because the mass distribution within the star is no longer spherically symmetric. There is a strong quadrupole component in the mass distribution. Fortunately, there is an exact solution of the GR equations for the case when the mass distribution has a quadrupole component. Using this solution, Sibgatullin \& Sunyaev (2000) plotted the dependence of the energy release due to the accretion onto a neutron star as a function of the rotation frequency of that star (Fig. 3). The existing GR solution permits us to find the efficiency of the energy release only in the case when the spin directions of the neutron star and accretion disk are parallel or anti-parallel. Unfortunately, the problem with an arbitrary angle between the axes of rotation of the neutron star and the accretion disk is much more complicated. In Figure 3 the positive values of the rotational frequency $f$ correspond to the case of corotation and negative values describe the case of counterrotation. The figure presents the computations for the equation of state (EOS) FPS in the classification of Lorenz et al. (1993) for a gravitational mass of the neutron $\operatorname{star} M=1.4 \mathrm{M}_{\odot}$.

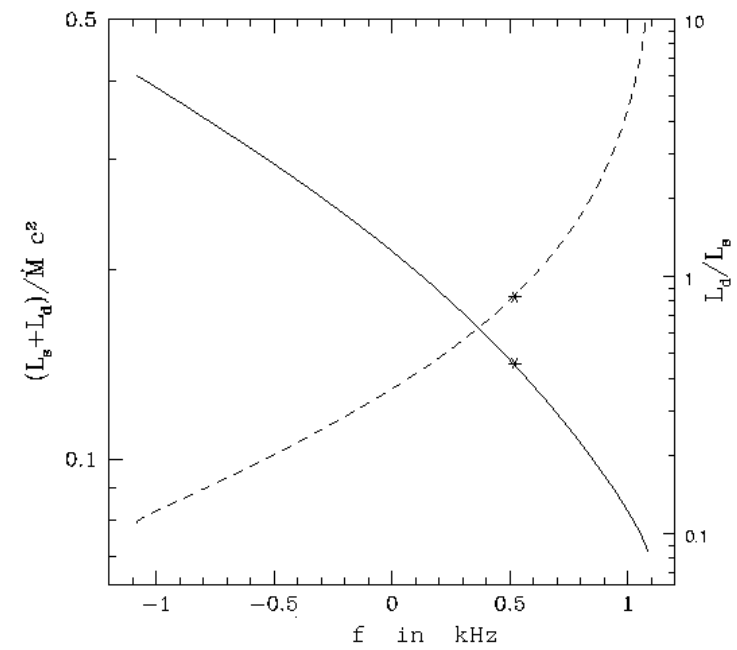

Fig. 3. Efficiency of the energy release in the disk $\left(L_{d}\right)$ and on the stellar surface $\left(L_{s}\right)$ as a function of the stellar rotation frequency $f$. Negative $f$ values correspond to the case of counter rotation of disk and star. The solid line and the numbers on the left axis give the value $\left(L_{s}+L_{d}\right) / \dot{M} c^{2}$. The dashed line and the values on the right axis give the ratio $L_{d} / L_{s}$. A gap between the marginally stable orbit and the stellar surface exists in the region leftside of the two asterisks on the solid and dashed curves. There is no such gap in the case of rapid corotation of star and disk $(f>550 \mathrm{~Hz})$. Adapted from Sibgatullin \& Sunyaev (2000). 
We see that the energy release efficiency drops rapidly with increasing frequency in the case of corotation and increases rapidly towards high frequencies of counter rotation. The ratio of the disk luminosity to the luminosity in the boundary layer or in the spreading layer near the surface of the star also strongly depends on the frequency of rotation. It is close to 1 for the case of corotation with $f=600 \mathrm{~Hz}$ and decreases up to 0.2 in the case of counter rotation with the same frequency.

The asterisks in Figure 3 give information on the existence of a gap between the marginally stable orbit in the accretion disk and the radius of the star. For frequencies of corotation higher than $550 \mathrm{~Hz}$ such a gap does not exist; then the disk is in contact with the surface of the neutron star. For lower frequencies of corotation and in the case of counter rotation for the EOS FPS and $M=1.4 \mathrm{M}_{\odot}$ there is a gap $R_{m}-R_{*} \approx$ $\left[1.44-3.06(f / \mathrm{kHz})+0.843(f / \mathrm{kHz})^{2}+0.6(f / \mathrm{kHz})^{3}-0.22(f / \mathrm{kHz})^{4}\right] \mathrm{km}$. In the most interesting case of corotation the gap is very narrow and the thickness of the boundary layer or the hight of the spreading layer usually exceeds the dimension of the gap. However, in the case of counter rotation (negative values of $f$ ) the gap could be sufficiently large that it has to be taken into account.

The energy release efficiency due to accretion onto a counter-rotating neutron star may reach very large values up to $0.67 \dot{\mathrm{M}} c^{2}$ for the case of a neutron star with baryonic mass $m=2.1 \mathrm{M}_{\odot}$ for $f=1.5 \mathrm{kHz}$ and the EOS FPS. Obviously, such a high energy release efficiency is connected with the spin down of the rapidly (counter) rotating star. This efficiency is much higher than that of disk accretion onto a Kerr black hole. In the case of corotation the energy release efficiency, due to accretion onto a Kerr black hole, is higher than in the case of counterrotation. This is reversed in the case of accretion onto a neutron star .

\section{Structure of the Boundary Layer}

The problem of disk accretion onto a neutron star without a magnetic field is two-dimensional. The height of an accretion disk at low accretion rates and luminosities $\left(0.01<L / L_{\mathrm{Edd}}<0.3\right)$ is small in comparison with the radius of the neutron star. Here and below $L_{\mathrm{Edd}}=\frac{4 \pi G M m_{p}}{\sigma_{T}}$ is the critical Eddington luminosity. The angular rotation frequency $\Omega^{\sigma_{T}}$ in the disk is close to keplerian and increases when matter approaches the neutron star. In the boundary layer the matter velocity must decrease to the velocity of rotation at the neutron-star surface and then matter must be redistributed over its equipotential surface. This surface is defined by the common influence of gravity and centrifugal forces. It is obvious that there must be a ring where $\Omega$ reaches its maximum, $d \Omega / d R=0$. There are two possible approaches to consider the matter flow beyond this point. We could assume that the boundary layer is described by the same equations as those valid for the 
accretion disk or we could consider the motion of matter in the spreading layer as belonging to the surface of the neutron star. We tried to investigate both of these approaches in one-dimensional approximations. In the paper by Popham \& Sunyaev (2000) we computed the structure and properties of the boundary layer considering it as a part of the disk. Figure 4 shows how the height of the boundary layer depends on the distance from the stellar surface for different accretion rates. In the case of a low accretion rate or $L \sim 0.01 L_{\mathrm{Edd}}$, the height of the disk in the "neck" between the accretion disk and the boundary layer is close to only 40 meters and the extension of the boundary layer about $1.5 \mathrm{~km}$. The situation drastically changes when we go to the case of high accretion rates with a luminosity close to the critical Eddington luminosity. The height of the neck between the boundary layer and the accretion disk in this case exceeds $2 \mathrm{~km}$ and the boundary layer extends up to 2 neutron-star radii.

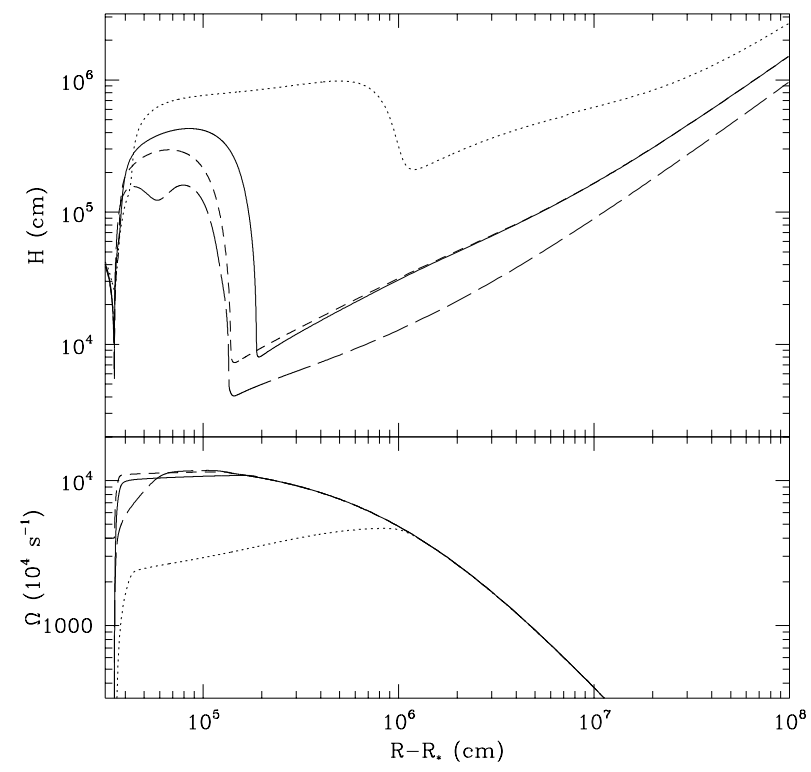

Fig. 4. The vertical pressure scale height $H$ (top) and the angular velocity $\Omega$ (bottom), for solutions with $\dot{M}=10^{-10}$ (long dash), $10^{-9}$ (solid) and $10^{-8} M_{\odot} y r^{-1}$ (dotted), all for a non-rotating neutron star, and for $\dot{M}=10^{-9} M_{\odot} y r^{-1}$ and a neutron star rotation frequency $f_{*}=636 \mathrm{~Hz}$ (dashed), all with standard viscosity and $\alpha=0.1$. Note the very small values of $H$ at the "neck" between the disk and the boundary layer in the lower $\dot{M}$ solutions, and the rapid increase in $H$ in the boundary layer. Adapted from Popham \& Sunyaev (2000). 
A more natural approach was considered by Inogamov \& Sunyaev (1999). This approach uses the shallow water or hydraulic approximation. It assumes that the thickness of the spreading layer on the surface of the neutron star is less than the circumference of the neutron-star equator $H<<2 \pi R_{*}$. This approach assumes that matter entering the equatorial ring with a very high rotational velocity of the order of $0.5 c$, where $c$ is the velocity of light. Then the matter begins to spiral slowly towards the poles losing its kinetic rotation energy due to turbulent friction with the dense underlying layer (Fig. 5 and Fig. 6).
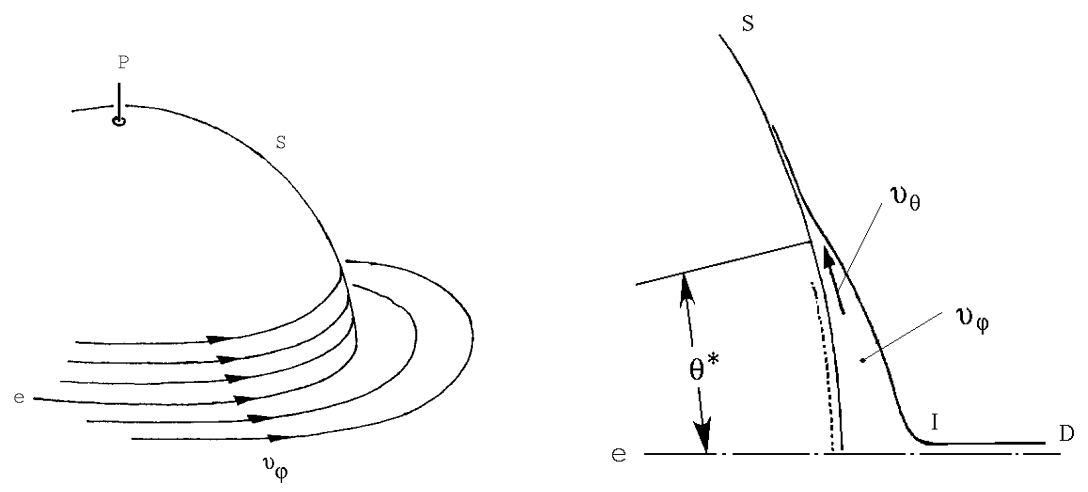

Fig. 5. Rotation of matter in the disk and on the stellar surface in the spreading layer model; $S$ is the stellar surface, $P$ is the pole, and $e$ is the equator.

Fig. 6. Spreading of the rotating plasma from the disk, $D$, over the neutron star surface, $S$. Here, $I$ is the intermediate zone near the disk neck, $\theta^{*}$ corresponds to the position of the hot belt, and $\theta>\theta^{*}$ is the cold part of the spreading layer. The rotation velocity $v_{\phi}$ (filled circle) is directed along the normal to the plane of the figure. The slowly circulating dense underlying layers of matter beneath the spreading layer are indicated by the dashes. Both figures are adapted from Inogamov \& Sunyaev (1999).

The thickness of the spreading layer is highest in the vicinity of the equator and decreases towards the poles. This means that matter is moving down the hill under the influence of gravity, the centrifugal force and the light pressure force. The problem is extremely interesting. We are dealing with radiation dominated plasma when the radiation pressure strongly exceeds the matter pressure. The sound speed is close to $0.1-0.15 c$. Radiative viscosity is also much stronger than the viscosity of plasma. The solution of the set of hydrodynamic equations results in the following picture (see Inogamov \& Sunyaev 1999 for details). Two bright belts equidistant from the equator 
appear on the surface of the neutron star due to disk accretion. Figure 7 gives the distance of the bright belts from the equator for 4 luminosities of the neutron star: $0.01,0.04,0.20$ and $0.80 L_{\mathrm{Edd}}$. The distance from the equator increases with luminosity.

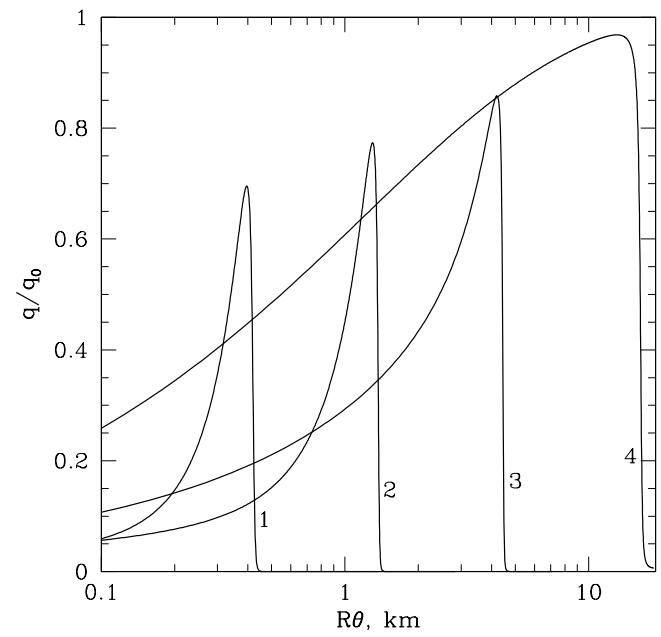

Fig. 7. Dependence of the surface brightness as a function of distance from the equator. Labels $1,2,3$ and 4 refer to $L_{\mathrm{SL}} / L_{\mathrm{Edd}}=0.01,0.04,0.2$, and 0.8 , respectively; $q_{o}$ is the critical Eddington flux. Adapted from Inogamov \& and Sunyaev (1999).

The energy release in the vicinity of the equator is very low because there centrifugal forces compensate gravity with high precision. Therefore, any substantial radiation flux could destroy the structure of the thin spreading layer. Fortunately, advection takes the radiation energy density and transports it to the bright belts above and below the equator (Fig. 8).

In these bright belts the rotational velocity of the spreading matter becomes low enough to permit the existence of a large radiation flux comparable to the critical Eddington flux $q_{0}=\frac{m_{p} c^{3}}{2 \sigma_{T} R_{g}}\left(\frac{R_{g}}{R_{*}}\right)^{2}=10^{22} \frac{\mathrm{W}}{\mathrm{m}^{2}}$, where $R_{g}$ is the gravitational radius. This flux value is comparable to radiation fluxes achieved in the most intense petawatt laser facilities (Perry 1996, Budil et al. 2000).

We are dealing here with a critical Eddington flux even in the case of a low luminosity of the neutron star $\left(0.01<L / L_{\mathrm{Edd}}<1\right)$. The surface of the bright belts is small and the high radiation flux from the narrow belts is consistent with the low luminosity of the star. 

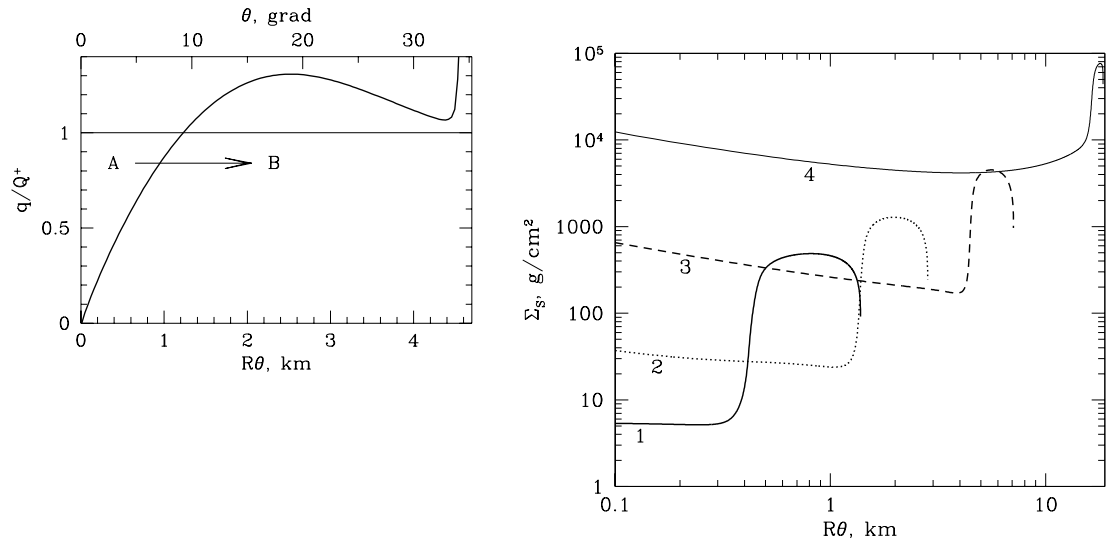

Fig. 8. The dynamics of the spreading layer is determined in many ways by the hydrodynamic transfer of radiative energy. The ratio of the energy flux, $q$, emitted per unit area of the radiating layer to the frictional energy release $Q^{+}$per unit area of the contact surface between the spreading layer and the star. The energy is transferred from zone A into zone B through meridional advection. This calculation is for $L_{\mathrm{SL}} / L_{\mathrm{Edd}}=0.2$.

Fig. 9. Column density of matter in the spreading layer as a function of the distance from the equator. A strong increase of $\Sigma$ occurs when the flow cools down and begins to move very slowly. Bright belts correspond to the regions of minimal $\Sigma$. Labels $1,2,3$ and 4 refer to $L_{\mathrm{SL}} / L_{\mathrm{Edd}}=0.01,0.04,0.2$ and 0.8 , respectively. Both figures are adapted from Inogamov \& Sunyaev (1999).

The matter in the spreading layer is practically levitating. The difference between the gravitational force and the centrifugal- and radiation pressure force is close to $(1-3) \times 10^{-3}$ of gravity. At higher longitudes the rotational velocity of matter and the velocity of the flow along the meridian decreases and the flow becomes subsonic, cool, dense and very slow.

One of the most interesting predictions of the theory of the spreading layer is the strong dependence of the matter column density in the spreading layer on the accretion rate or the luminosity of the neutron star (see Fig. 9). In the case of a low luminosity the levitating layer in the bright belts is optically thin against Thompson scattering $\tau_{T} \sim 2$. Under these circumstances it is impossible to radiate the energy released due to accretion at low temperatures. Comptonization forms hard tails. In the case of a high luminosity the bright belt has a large column density (up to $10 \mathrm{~kg} / \mathrm{cm}^{2}$ ). Then free-free processes and comptonization form Bose-Einstein type spectra inside the spreading layer and the resulting spectrum is much softer than in the case of low luminosity. 


\section{Time Variability in the Accretion Disk and in the Boundary Layer}

All instabilities existing in the accretion disk modulate the flow of matter onto the neutron-star surface. Therefore, we could expect that the majority of the types of variability we observe in accreting black holes must manifest themselves in accreting neutron stars with characteristic timescales proportional to the mass of the accreting object (see e.g. Shakura \& Sunyaev 1976, Wijnands \& van der Klis 1999). The spreading layer on the surface of the neutron star is the source of additional high-frequency instabilities (see the discussion in Sunyaev \& Revnivtsev 2000). Their origin is obvious - the matter in the bright belts is radiation dominated, levitating, the height is smaller than in the region of the main energy release in the accretion disk, the sound velocity is huge and corresponding sound frequencies are very high.

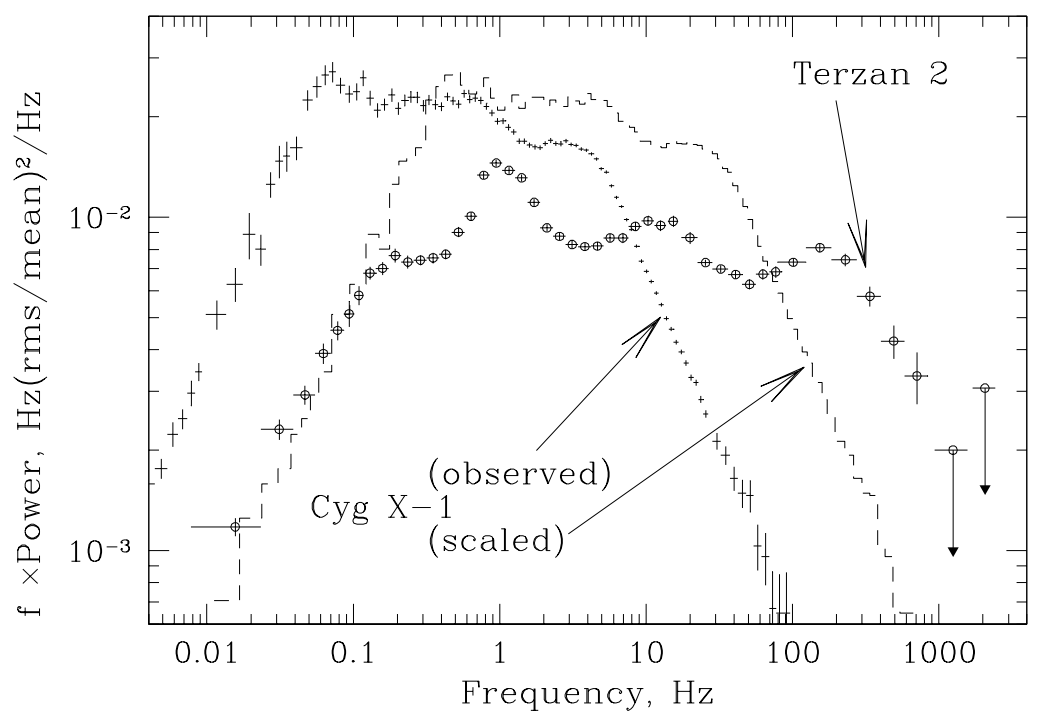

Fig. 10. Comparison of the power spectra of a black hole (Cyg X-1) and a neutron star (Terzan 2). The dashed line shows the power spectrum of Cyg X-1 scaled according to the mass ratio with Terzan $2\left(f_{\mathrm{CygX}-1} \times 7 \rightarrow f_{\mathrm{CygX}-1}^{\text {scaled }}\right.$. This simple scaling is important but insufficient to explain fully the difference in the high frequency variability of Terzan' 2 and Cyg X-1. Note that the slopes of the power density spectra of Terzan 2 and Cyg X-1 in the high and low frequency limits are similar, but that the power spectrum of Terzan 2 is significantly broader than that of Cyg X-1. Adapted from Sunyaev \& Revnivtsev (2000).

Sunyaev \& Revnivtsev (2000) compared the power density spectra of 9 black holes and 9 neutron stars observed by RXTE in their low/hard state. 
There is a very strong difference. In the power density spectra of accreting neutron stars with a weak magnetic field significant power is contained at frequencies close to one $\mathrm{kHz}$. At the same time, most Galactic accreting black holes demonstrate a strong decline in the power spectra at the frequencies higher than 10-50 Hz. In principle this might open an additional way to distinguish the accreting neutron stars from black holes in X-ray transients (we do not mention in this paper the well-known differences: X-ray bursts or $\mathrm{X}$-ray pulsations). Fig. 10 compares the power density spectrum of Cyg X-1 with the power density spectrum of the X-ray burster in Terzan 2.

The simplest assumption is that the characteristic frequencies in the power spectra of the sources scale as $\mathrm{M}^{-1}$ (Shakura \& Sunyaev 1976). This scaling law is valid for e.g. the keplerian frequency in the vicinity of the marginally stable orbit, the thermal and secular instabilities of the accretion disk in the region of main energy release, and the Balbus-Hawley instability. However, this assumption does not account for the observed difference in the high frequency variability between neutron stars and black holes.

\section{References}

1. Bolton C.T. (1972) Nature 235, 271

2. Budil, K. S., Gold, D. M., Estabrook, K. G., Remington, B. A., Kane, J., Bell, P. M., Pennington, D. M., Brown, C., Hatchett, S. P., Koch, J. A., Key, M. H. and Perry, M. D. (2000) ApJ Suppl 127, 261-265

3. Galeev, A., Rosner, R., Vaiana, G. (1979) ApJ, 229, 318

4. Gilfanov, M., Churazov, E., Revnivtsev, M. (2000) MNRAS 316, 923

5. Inogamov, N., Sunyaev, R., (1999) Astr. Letts 25, 269, astro-ph 9904333

6. Lorenz, C. P., Ravenhall, D. G., Pethick, C. J. (1993) Phys. Rev. Lett. 70, 379

7. Lyuty, V., Sunyaev, R., Cherepashchuk, A. (1973) Soviet Astronomy 50, 3

8. Narayan, R., Yi, I. (1995) ApJ 452, 710

9. Perry, M. D. (1996) Sci. Tech. Rev. 12, 4

10. Popham, R., Sunyaev, R. (2000) ApJ (in press), astro-ph 0004017

11. Sibgatullin, N., Sunyaev, R. (2000) Astr. Letts 26, in press

12. Shakura N., Sunyaev, R. (1973) Astron. Astrophys. 24, 337

13. Shakura N., Sunyaev, R. (1976) MNRAS 175, 613

14. Strohmayer, T., Zhank, J. H., White, N. E., Lapidus, I. (1998) Astrophys.J. 498, 1358

15. Sunyaev, R., Revnivtsev, M. (2000) Astronomy and Astrophysics 358, 617

16. Sunyaev, R., Titarchuk, L. (1980) Astron. Astrophys 86, 121

17. Sunyaev, R., Titarchuk, L. (1989) Proceedings of the 23rd ESLAB Symposium 1,627

18. Sunyaev, R, Trümper, J. (1979) Nature 279, 506

19. Tanaka, Y., Shibazaki, N. (1996) ARA\&A 34, 607

20. Tananbaum, H., Gursky, H., Giacconi, R., Jones, C. (1972) ApJ 177, L5

21. Wijands, R., van der Klis, M. (1999) ApJ 514, 939 